\title{
Testing a prototype rotary mechanism for GMTIFS
}

I. Price, J. Hart, E. O'Brien, R. Sharp, G. Gausachs, et al.

I. Price, J. Hart, E. O'Brien, R. Sharp, G. Gausachs, G. Bloxham, C. Vest, J. Gilbert, B. Espeland, "Testing a prototype rotary mechanism for GMTIFS," Proc. SPIE 10706, Advances in Optical and Mechanical Technologies for Telescopes and Instrumentation III, 107063M (10 July 2018); doi: $10.1117 / 12.2312337$

SPIE Event: SPIE Astronomical Telescopes + Instrumentation, 2018, Austin, Texas, United States 


\title{
Testing a prototype rotary mechanism for GMTIFS
}

\author{
I. Price ${ }^{\mathrm{a}}$, J. Hart ${ }^{\mathrm{a}}$, E. O’Brien ${ }^{\mathrm{a}}$, R. Sharp ${ }^{\mathrm{a}}$, G. Gausachs ${ }^{\mathrm{a}}$, G. Bloxham ${ }^{\mathrm{a}}$, C. Vest $^{\mathrm{a}}$, J. Gilbert ${ }^{\mathrm{a}}$, \\ and B. Espeland ${ }^{\mathrm{a}}$ \\ ${ }^{a}$ Research School of Astronomy and Astrophysics, Australian National University, Canberra, \\ ACT 2611, Australia
}

\begin{abstract}
The GMTIFS instrument requires multiple rotary mechanisms that will operate in a cryogenic environment. Angular precision up to one arc-second is required without the use of IR sources as part of an encoder. A general design that uses an annular conical rim bearing supported by three pairs of tapered pinch rollers has been proposed. One pair of pinch rollers is mounted on a flexure hinge to provide preload and accommodate thermal expansion. A pair of offset cylindrical cams carried by the rotor, and four capacitive distance sensors fixed to the stator are utilized to implement a resolver. This provides a measure of the rotor orientation that is insensitive to runout of the rotor. A prototype of this design was constructed and tested in the lab to investigate the effect of runout in the tapered rollers and assess the performance of the rim bearing and various resolver designs. We present the results of this testing.
\end{abstract}

Keywords: Resolver, Rim Bearing, Cryogenic, Capacitive, Runout

\section{INTRODUCTION}

The next generation of infrared instruments designed for extremely large telescopes have challenging requirements for rotary mechanisms. In the specific case of the GMTIFS instrument one mechanism requires sub-arcsecond precision and stability on the timescale of a few hours ${ }^{1} .^{2}$ Other mechanisms have lower precision requirements but must support transmissive optics. Coupling rotary encoders to a mechanism via a gear train can increase angular resolution, but this does not imply increased precision, and in the case of small mechanisms the precision is limited by bearing runout. The cryogenic environment and the need to limit electrical noise, thermal emission and the risk of light leaks from internal IR sources effectively prohibit the use of conventional encoders. A general purpose resolver based on capacitive sensors has been designed that overcomes those issues. ${ }^{3}$ The principle is based on a pair of cylindrical cams that are fixed to the rotor and four capacitive sensors fixed to the stator that measure the distance to the cams. The pair of cams is structured such that their centers are displaced from the rotation axis by equal distances in opposite directions. This can be arranged by having the cams in parallel planes or in a single plane with different radii. The sensors are arranged in opposing pairs on orthogonal axes, normal to the rotation axis. One sensor in each opposing pair measures the distance to one cam and the other sensor the distance to the other cam. This structure allows systematic errors due to bearing runout to be cancelled.

A rotary mechanism has been designed that uses three pairs of pinch rollers that contact with a double coned annular rotor. The pinch rollers are tapered to match the cones on the rotor. Two of these pairs are fixed and the third pair are carried on a flexure mount. The flexure provides preload to support the rotor under a changing gravity vector, and to accommodate thermal expansion. The cams from the resolver can also have an annular structure, which allows the rim-bearing and resolver to be directly coupled into a rotary mechanism.

A prototype mechanism was constructed around the rim-bearing design with integral resolver. This is not representative of any particular mechanism in the GMTIFS instrument, but it allows the resolver principle and rim-bearing to be tested.

Further author information: (Send correspondence to Ian Price.)

Ian Price.: E-mail: ian.price@anu.edu.au

Rob Sharp: E-mail: rob.sharp@anu.edu.au

Advances in Optical and Mechanical Technologies for Telescopes and Instrumentation III, edited by Ramón Navarro, Roland Geyl, Proc. of SPIE Vol. 10706, 107063M · C 2018 SPIE CCC code: $0277-786 \mathrm{X} / 18 / \$ 18 \cdot$ doi: $10.1117 / 12.2312337$

Proc. of SPIE Vol. 10706 107063M-1 
The analytic principle of the resolver is described in $\S 2$, the sources of error that limit the performance are discussed in $\S 3$, we describe the prototype and the test system in $\S 4$, present the results and evaluate the performance in $\S 5$.

\section{RESOLVER}

The resolver geometry is depicted in figure 1. The scales have been greatly exaggerated to illustrate the working principle. The proposed practical designs have a small cam offset, $r$, and a large cam radius, $R$. The former is constrained by the dynamic range of capacitive sensors and will typically be less than $1 \mathrm{~mm}$. We define a Cartesian coordinate system fixed in the stator, with the $\mathrm{X}$-axis passing through sensor number 1, the $\mathrm{Y}$-axis passing through sensor number 2 , and $\mathrm{Z}$ the ideal rotation axis. The sensors are equidistant from the $\mathrm{Z}$-axis and located on the X-Z and Y-Z planes. They are aligned such that they measure distances in either the X or Y direction. The distance to the nearest point on the cam in the direction of measurement is $d_{i}$. The expressions for sensors 1 to 4 are

$$
\begin{aligned}
d_{1} & =C_{1}-x-r \cos (\theta), \\
d_{2} & =C_{2}-y-r \sin (\theta), \\
d_{3} & =C_{3}+x-r \cos (\theta), \\
d_{4} & =C_{4}+y-r \sin (\theta),
\end{aligned}
$$

where $C_{i}$ are a constants that depends on the sensor location and the cam radius. Due to the symmetrical structure of the rotor and cam assembly, the expression for opposing sensors differs only in the sign of the rotor runout term. Adding Eqns (1) and (3), and Eqns (2) and (4) eliminates $x$ and $y$. Dividing the resulting expressions by two and rearranging gives the sin and cosine of the rotor angle in terms of the average gaps in the $\mathrm{X}$ and $\mathrm{Y}$ directions,

$$
\begin{aligned}
& \cos \theta=\left(\frac{1}{r}\right)\left(\frac{C_{1}+C_{3}}{2}-\frac{d_{1}+d_{3}}{2}\right), \\
& \sin \theta=\left(\frac{1}{r}\right)\left(\frac{C_{2}+C_{4}}{2}-\frac{d_{2}+d_{4}}{2}\right) .
\end{aligned}
$$

The ratio of Eqns (5) and (6) eliminates $r$ and the arctangent of the ratio yields the rotor angle.

In figure 1 the radii of the two cams is the same, but they is no requirement for this to be the case to eliminate the runout from the expression for the rotor angle. This provides considerable freedom for practical designs. The cam de-center term, $r$, was eliminated because the two cams are displaced equal distances in opposite directions. While this may seemingly require tight manufacturing tolerances for real devices, it is not the case. The resolver treats the midpoint between the cam centers as the center of rotation of the mechanism. This is a fixed point in the rotor, but it need not coincide with the rotor axis. Misalignment between the resolver's center and the rotor's center merely leads to systematic sinusoidal components in the runout signature, without directly impacting the resolver performance.

Being able to derive the rotor angle in a way that is insensitive to runout is essential for the construction of precision rotary mechanisms. The resolver principle outlined is insensitive to runout but is reliant on precision measurement of small gaps. This is commonly the domain of capacitive sensors.

\section{SOURCES OF ERROR}

It is convenient to express the right hand side of Eqns (5) and (6) as the dimensionless quantities, $G_{x}$ and $G_{y}$ respectively, which are simply a linear transformation of the average gaps. The expression for the rotor angle therefore becomes $\tan \theta=\frac{G_{y}}{G_{x}}$. Taking the derivative and substituting $G_{x}=\cos \theta$ and $G_{y}=\sin \theta$ gives

$$
\delta \theta=\cos \theta . \delta G_{y}-\sin \theta . \delta G_{x},
$$

which characterizes the error in deriving the rotor angle. It follows that angular precision is limited by the ratio of sensor precision to the cam de-center $\left(\delta \theta \sim \frac{\delta d}{r}\right)$. Devices requiring sub arcsecond precision therefore require 


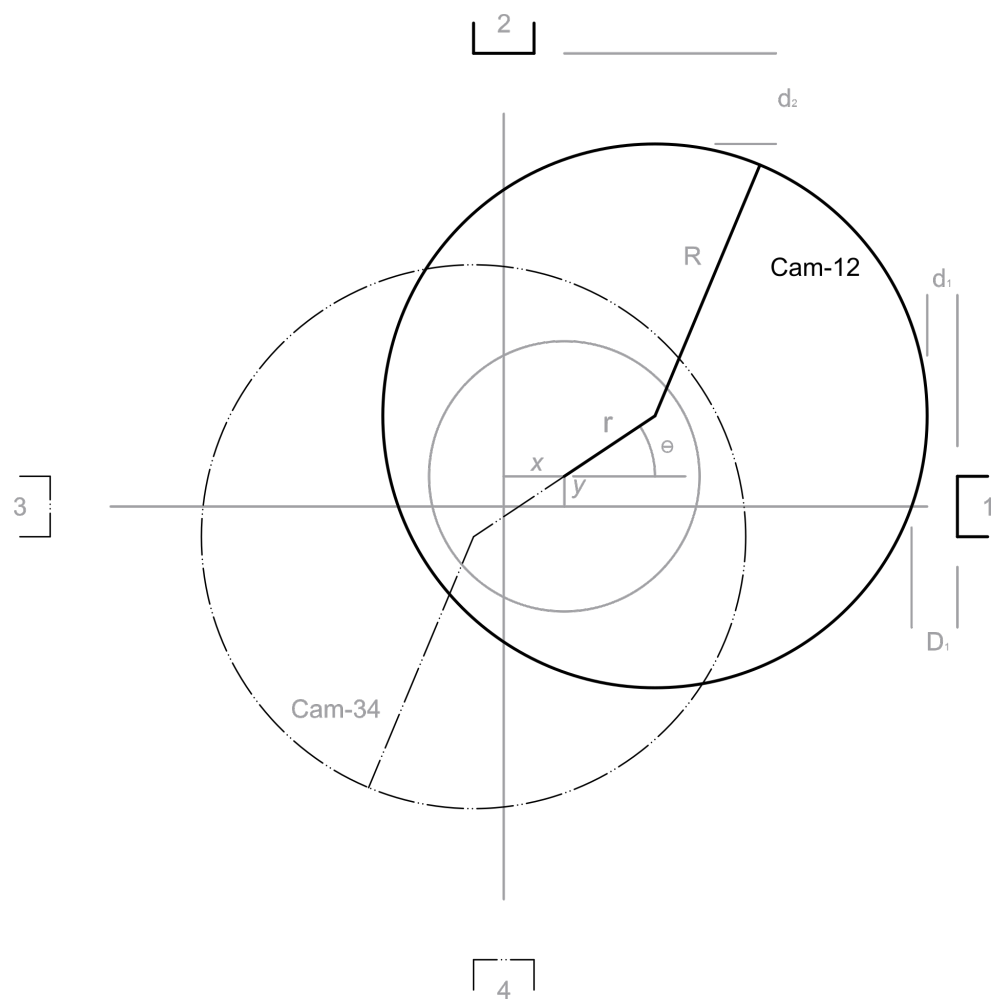

Figure 1. The center of the rotor is displaced from the origin to $(x, y)$ due to bearing runout. The capacitive sensors are fixed to the stator at points 1,2, $3 \& 4$ and measure the distance to a cylindrical cam. Cam-12 is monitored by sensors 1 $\& 2$ and Cam-34 is monitored by sensors $3 \& 4$. The cams are de-centered from the rotor axis by $r$ in opposite directions. The rotor has been rotated from the reference line by an angle $\theta$. The cams have radius $R$. In the ideal system the sensors would measure the distance along the sensor's axis to the plane tangent to the cam and parallel to the sensor face, $d_{i}$. In reality the sensor measures the distance $D_{i}$ with systematic errors resulting from the cam curvature and the tilt of the target relative to the sensor face.

sensors that have at least 18-bit resolution and random errors less than 1 part in 207000 over the full dynamic range.

Errors in the calibration constants will also contribute to $\delta G_{x}$ and $\delta G_{y}$, but these will be systematic. As such they affect the absolute accuracy of the resolver, though the error is a stable function of the rotor angle. Changes in the sensor positions or the cam radius as a result of thermal expansion or mechanical stress manifest as calibration errors. Thermal expansion of the stator, rotor or both change the nominal sensor-cam gaps and apply the same bias to both $\delta G_{x}$ and $\delta G_{y}$. From Eqn (7) we see that this has no effect at $\theta=45^{\circ} \& 225^{\circ}$, and the largest effect at $\theta=135^{\circ} \& 315^{\circ}$. It can be shown that $\delta G_{i} \approx k \delta T$, where $k$ is the CTE of the stator and $\delta T$ is the change in temperature, because $C_{i} \approx r$ in order to utilize the full dynamic range of the sensors. The effect of thermal expansion is therefore dependent only on the material properties, not on the dimensions. For a resolver constructed from an aluminium alloy, oriented at $\theta=45^{\circ}$, the sensitivity to temperature change, $\frac{\delta \theta}{\Delta T}$, is $7 \mathrm{arcsec} / \mathrm{K}$.

The derivation of the runout invariant resolver is based on measuring distances to the high point on the cam. The intrinsic cam de-center means the high point is rarely aligned to the center of the sensor. In general the sensor-axis aligned distance is slightly larger and the tangent plane to the cam at that point is not parallel to the face of the sensor. Moreover, the curvature of the cam concentrates the field lines and biases the distance measure. Models for the distance measurement error for target tilt and curvature are non-linear. The former is 
given by

$$
\Delta D=D\left[\frac{1}{1+\frac{R_{s}^{2}}{4 D^{2}} \tan ^{2} \phi}-1\right],
$$

where $D$ is the actual distance between the sensor and the target at the point on the sensor axis, $R_{s}$ is the radius of the active region of the sensor and $\phi$ is the tilt of the target relative to the sensor face. This shows that capacitive sensors underestimate the distance to tilted targets and the closer the target the greater the underestimate. Though non-linear, $\frac{\Delta D}{D}$ is small and $D$ and $\phi$ can be solved iteratively given the resolver geometry and a reasonable initial guess for $\theta$. The uncorrected distance measure and Eqns (5) and (6) provide an excellent initial estimate.

Bearing runout translates the rotor center, but it can also lead to tip-tilt of the rotation axis. This also leads to tip-tilt of the cylindrical cams in the resolver target, and changes in the sensor-target distances. If the sensors and cams are all in the same plane then the gaps measured by opposing sensors change by the same amount,

$$
\Delta D \approx R\left(1-\frac{1}{\cos \psi}\right),
$$

where $\psi$ is the component of the rotor axis tilt in the direction of the sensor. This results in an error in the corresponding average gap, but the change is likely to be small unless the tilt is large. However, if the cams are in different planes then $\Delta D \approx h \cdot \sin \psi$, where $h$ is the distance between the two planes.

\section{PROTOTYPE TEST SYSTEM}

A prototype rotary mechanism was designed to evaluate both the resolver and a rim-bearing rotor suitable for supporting transmissive optics in a cryogenic environment. The stainless steel rotor is $100 \mathrm{~mm}$ in diameter with a $50.8 \mathrm{~mm}$ bore, is $10 \mathrm{~mm}$ thick and has two conical surfaces at the rim. Two different resolver targets were designed. These are separate parts machined from 6061 aluminium. Mating surfaces on the rotor and target ensure nominal alignment to minimize systematic runout. One of the resolver targets has two cylindrical cams located in parallel planes. Each cylinder is $12 \mathrm{~mm}$ long and has a radius of $66 \mathrm{~mm}$. They are separated by by $4 \mathrm{~mm}$. The other resolver target has two half-cylinder cams located in the same plane. This target has a continuous working range of just under $90^{\circ}$. The stator is a $16 \mathrm{~mm}$ thick 6061 aluminium plate that supports four stainless steel sensor posts and a rotor drive train. Two of the sensor posts each support a pair of tapered rollers that mate with the conical surfaces on the rotor. The cylindrical body of the capacitive sensors are fitted in one of two holes in the upper section of the posts and clamped in place. The holes in the posts position the active area of the sensors in the center of a target cam and direct the sensor axis towards the Z-axis. The sensors are Micro-Epsilon model CSH1(20)-CAm1,4 and have an extended $2000 \mu \mathrm{m}$ range. The cams are de-centered $950 \mu \mathrm{m}$, leaving a nominal $50 \mu \mathrm{m}$ clearance to accommodate bearing runout. The stator is designed for the sensors to be installed flush with the front face of the posts. For the two-plane target the sensors are placed in the upper holes on posts 1 and 2 and the lower holes in posts 3 and 4 . For the one plane target all sensors are in the lower holes. A third set of pinch rollers are supported on a flexure hinge between posts 3 and 4 . A stepper motor drives one of the rollers via a 1:1 pinion gear train. The flexure hinge provides a preload of approximately $60 \mathrm{~N}$ to seat the rotor against the pinch rollers. The stepper motor has $200 \mathrm{steps} / \mathrm{rev}$ and is driven by a Copley Controls Accelnet Plus TEL-090-07 EtherCAT driver. The driver is configured for 512 microsteps per motor step and open loop position control with trapezoidal motion profiles. The center of the rotor supports a reflective optic in one of two configurations. In the vertical configuration the optic surface is perpendicular to the $\mathrm{Z}$ axis and located above the resolver target and stator structures. This allows the rotor angle to be measured over a small angular range with an autocollimator to asses the resolver performance. In the horizontal configuration the optic is supported within the central bore of the rotor and the entire mechanism is tipped $90^{\circ}$. In this configuration tip and tilt of the rotor plane can be measured with an autocollimator to assess the performance of the rim-bearing. A schematic of the prototype rotary mechanism assembly in the two plane target and vertical optic configuration is shown in figure 2. The complete mechanical test assembly, including the autocollimator, is shown in figure 3 .

A Taylor-Hobson autocollimator and the rotary mechanism were mounted side-by-side on an optical table with the autocollimator pointed at the optic carried by the rotor. A PointGrey Grassshopper camera with 


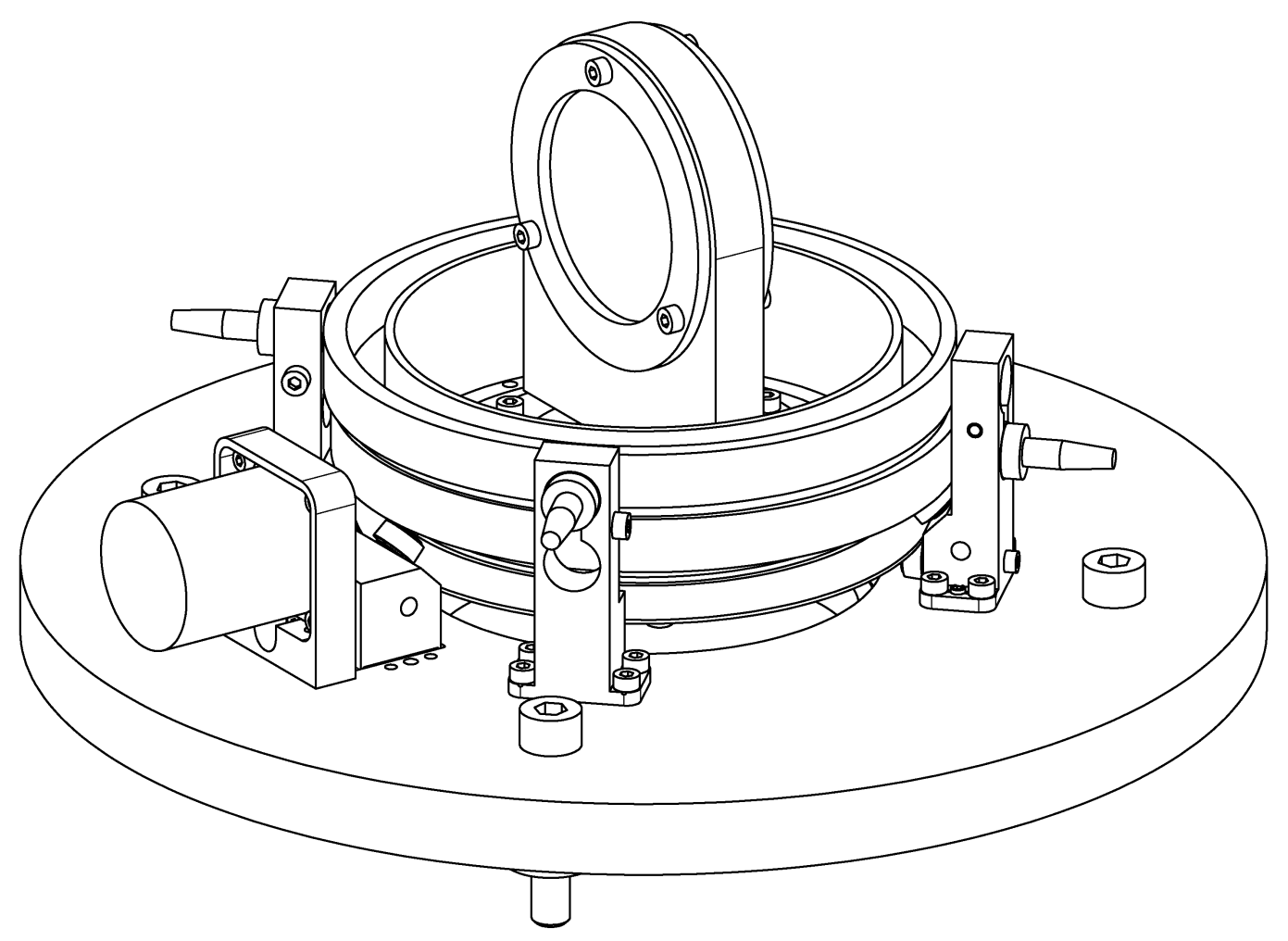

Figure 2. The rotary mechanism prototype assembly with a two-plane resolver target and the reflective optic in the vertical plane.

a $75 \mathrm{~mm}$ lens reimaged the projection of the reticle from the autocollimator. The location of the reticle was measure in pixel coordinates from a narrow strip extracted from the full image. The effective resolution of the optical system is approximately $0.3 \mathrm{arcsec} / \mathrm{pixel}$ and the error in the reticle position measure is less than 0.2 pixels. This arrangement provides a measure of the rotor angle over a one arcminute range to compare with the measurement from the resolver.

Each of the capacitive sensors is connected to a DL6530 unit within a DT6530 controller module. The EtherCAT interface was used to obtain the raw 24-bit distance measurement for each channel. Four PT100 temperature sensors were used to monitor the temperature of the system. Two were attached to the stator and one to the stepper-motor body. The remaining sensor measured the ambient air temperature. A single EtherCAT field bus coupled all of these components into a simple control and data acquisition system built on a client-server architecture. Experiment control, data acquisition and image analysis was orchestrated via shell scripts.

Development of automated test processes and the initial tests were conducted with the building heating, ventilation and air-conditioning system (HVAC) providing temperature control. This was clearly inadequate for assessment of the resolver precision, so the rotary mechanism and autocollimator were remounted to the optical table via stand-offs made from low thermal conductivity material and the entire system was enclosed in a insulated structure. The air in the enclosure was heated via a resistive load and variable current source, and circulated with fans. The heater current was regulated in closed-loop to stabilize the stator temperature at $30^{\circ} \mathrm{C}$. The set-point was approximately $6 \mathrm{~K}$ above the ambient temperature of the room which ensured some heating power was always required to maintain a stable stator temperature. The time constant for the enclosed thermal mass was approximately 2.75 hours. 


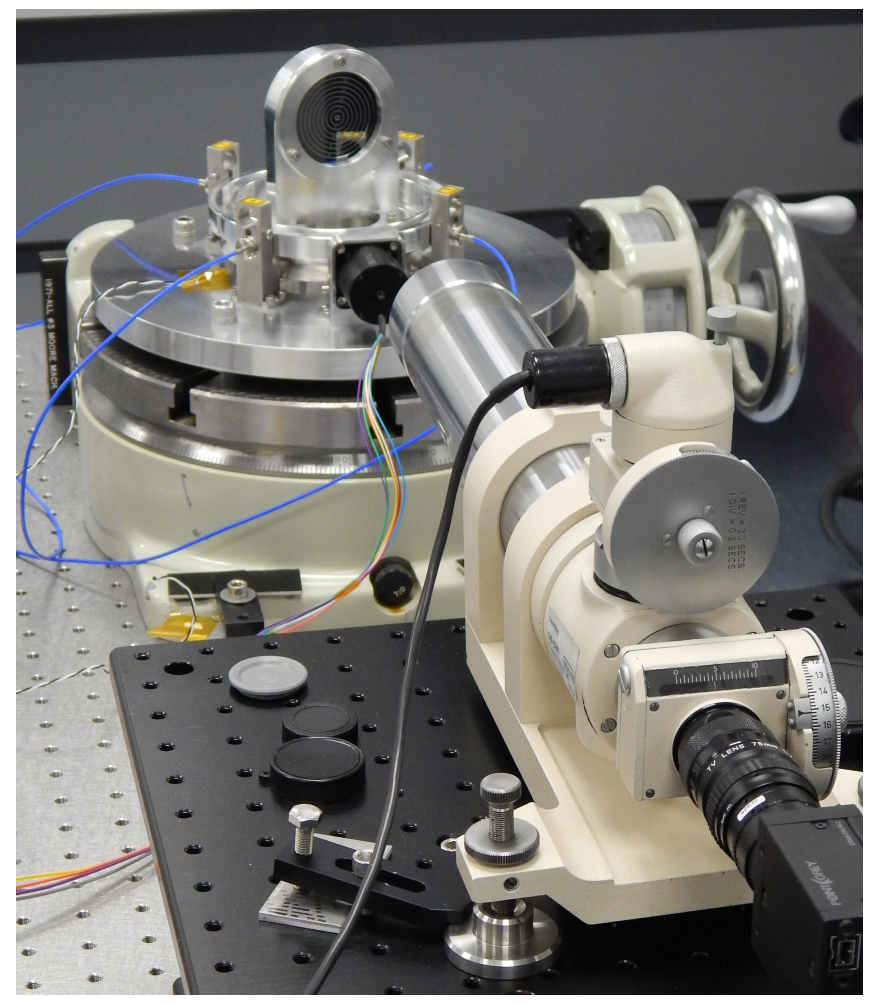

Figure 3. The test system configured for measuring the rotor angle with the resolver and autocollimator.

\subsection{Calibration}

The constants $C_{1}$ to $C_{4}$ were determined empirically. The rotor was driven through three rotations in 1000 microstep increments and the sensor measurements recorded at each step. Due to tracking errors in the rimbearing friction drive train the absolute motor position is only an approximate measure of the rotor angle, but the nominal drive ratio is $1.192 \times 10^{6} \mu$ steps/rev. For the two plane resolver target the function $f(p)=$ $A+B \cdot \sin (C \cdot p+D)$ was fitted to the data for each sensor, where $p$ is the motor position in microsteps and $A, B, C \& D$ are free parameters. The value for $A$ is the calibration constant $C_{i}$ for the sensor. For the one plane resolver target the half-cylinder target structure meant the calibration constants have greater uncertainty. More sophisticated methods of calibration are possible that take advantage of the runout cancelling nature of the resolver design, but given the objective of the testing was not absolute resolver precision this was not warranted.

\subsection{Scans}

To assess the performance of the resolver the rotor was slewed to project the reticle just outside of the field of view of the camera. The motor was then stepped in one microstep increments to scan the projected reticle across the field of view. After each step an image was captured and the reticle position was measured. The distance measures from the four sensors and the temperatures were also sampled. The resolver angle was then computed and all of these data were recorded. When the reticle reached the edge of the field of view the rotor was slewed by up to $180^{\circ}$ in either direction, then back to the starting position to repeat the cycle. All scans across the camera field of view were in the same direction and the sequence of slews at the start of the scan eliminated any backlash. Each scan took about 3 minutes.

Due to the small field of view of the optical system the relationship between the reticle position and the resolver angle was expected to be linear. Scanning, rather than servoing to a set-point, separates the resolver performance from gear errors, the microstepping size and backlash in the drive train. The duration of a single scan is also short compared to the thermal time constant of the system and are therefore virtually unaffected by thermal drift. 


\section{RESULTS}

Equations (1) to (4) are combined to eliminate the runout terms to derive the rotor angle. Therefore they can also be combined to measure the runout term. Figure 4 shows the $x$ and $y$ components of the rim-bearing runout from three rotations of the rotor. This shows periodic structure on the scale of one revolution and at scales 11.6 times smaller. This is not surprising, since the gear ratio between the rotor and the rollers is $11.6: 1$. The structure on the scale of one revolution is largely due to a $\approx 10 \mu \mathrm{m}$ misalignment of the resolver and rotor centers. The systematic effects of a tilted target and differences between $D_{i}$ and $d_{i}$ also contribute on this angular scale.

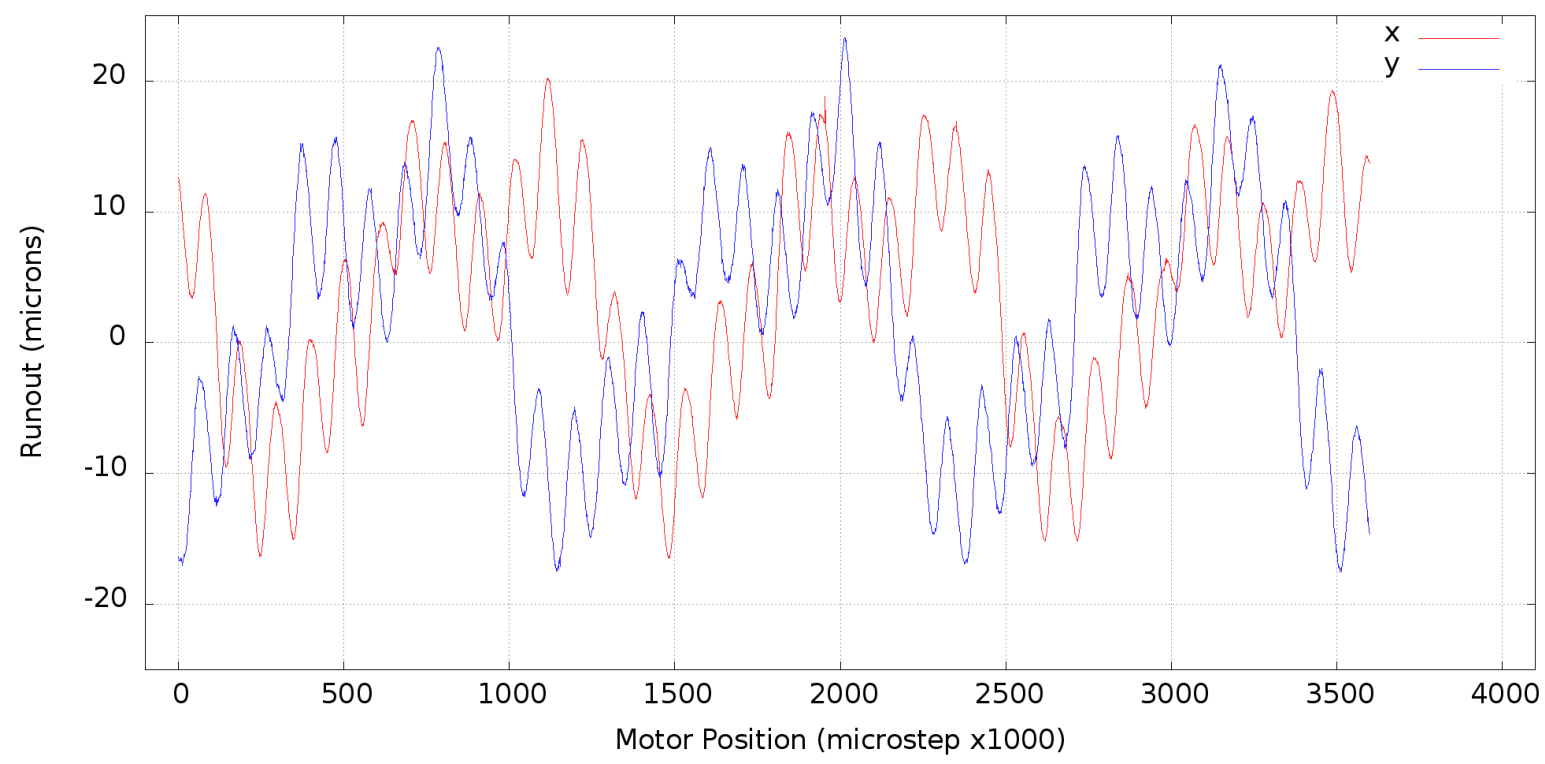

Figure 4. The $x$ and $y$ components of the rim bearing runout measured with the two-plane resolver target. Concentricity errors in manufacture of the tapered rollers produce the oscillation on the shortest scale. A range of factors contribute to the period structure on the scale of one rotation.

Initially the stator was oriented such that autocollimator would measure rotor angles near $\theta \approx 45^{\circ}$ and data were recorded for 175 scans. During this time the temperature of the immediate environment was controlled only with the building HVAC system. Figure 5 shows resolver angle versus reticle position with a separate trace for each scan. The broad band of lines in that figure show a change in the absolute measure of the rotor angle from scan to scan. However individual scans exhibit a linear relationship with low scatter. A straight line was fitted to each scan to obtain the resolver derived angle at a fixed optical reference angle. The RMS of the residuals was also computed. The deviation of the resolver angle from $44.26^{\circ}$ at the optical reference is plotted against scan number on the left of figure 6 . The RMS of the residuals to the linear fit is plotted on the right. There is clearly a drift over time in both the resolver derived angle and the residuals. The latter starts to increase after 80 scans and jumps sharply after 140 scans. The cause of this change is unknown. The time series in the left-most plot in figure 6 was high-pass filtered to remove the drift. The RMS of the filtered series is 1.7 arcsec, which characterizes the repeatability of the mechanism on short time scales. Note this is still more than three times larger than the variation in the scan with the largest residual.

The system was reconfigured for the one-plane resolver target and enclosed in a thermally isolated chamber. The stator temperature was regulated with the set-point at $30^{\circ} \mathrm{C}$. The building HVAC system maintained the local external environment around $24^{\circ} \mathrm{C}$. Since the temperature was stabilized, the stator was oriented such that the autocollimator could measure angles away from the temperature invariant points. Due to the limited range of resolver angle possible with the one-plane target a highly sensitive orientation was not possible. An orientation near $-108^{\circ}$ was chosen as the most sensitive orientation possible that kept the sensors clear of the discontinuity in the resolver target between the two half-cylinders. The deviation of the stator temperature from 


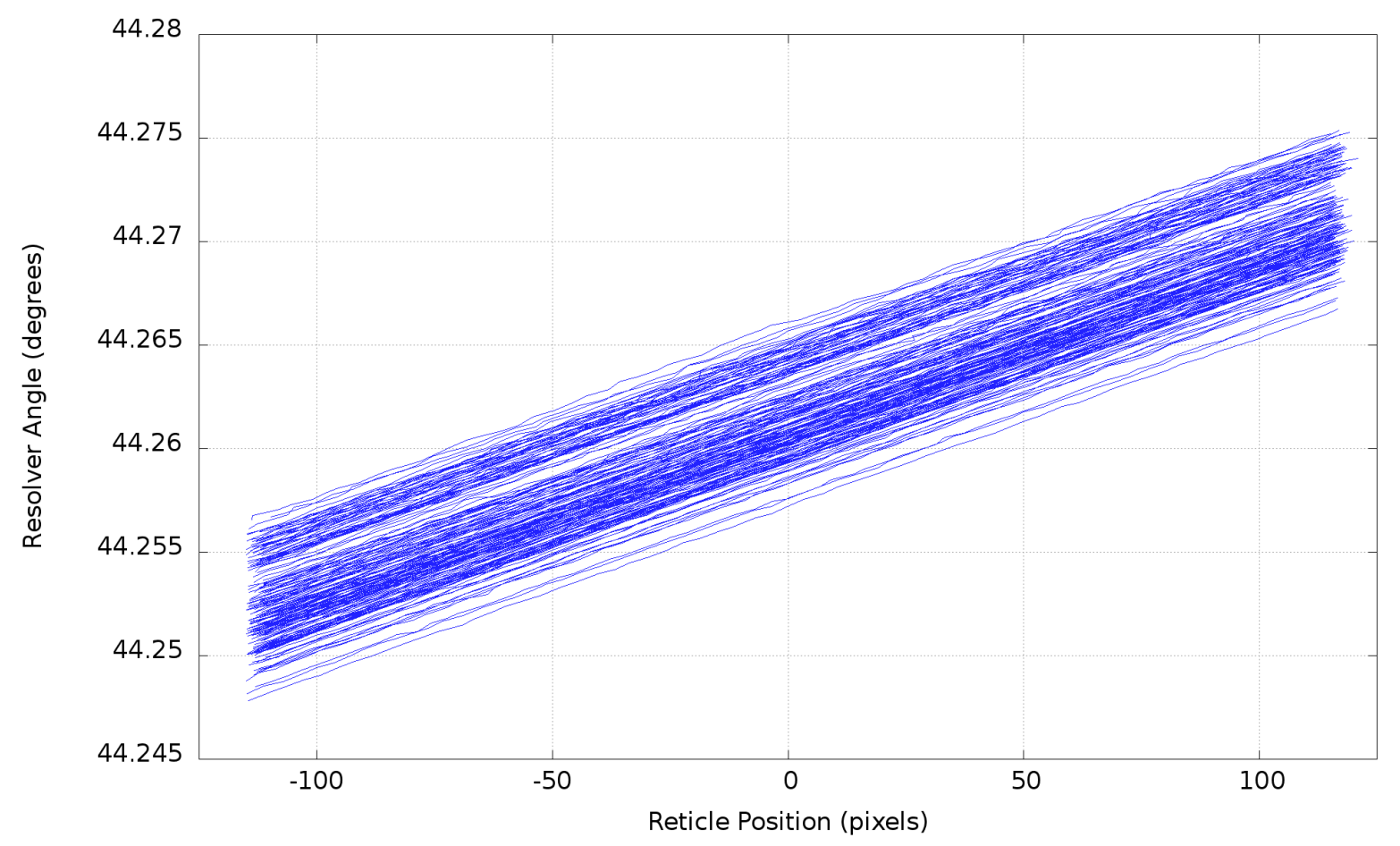

Figure 5. Scans of two plane resolver with rotor angle 45.
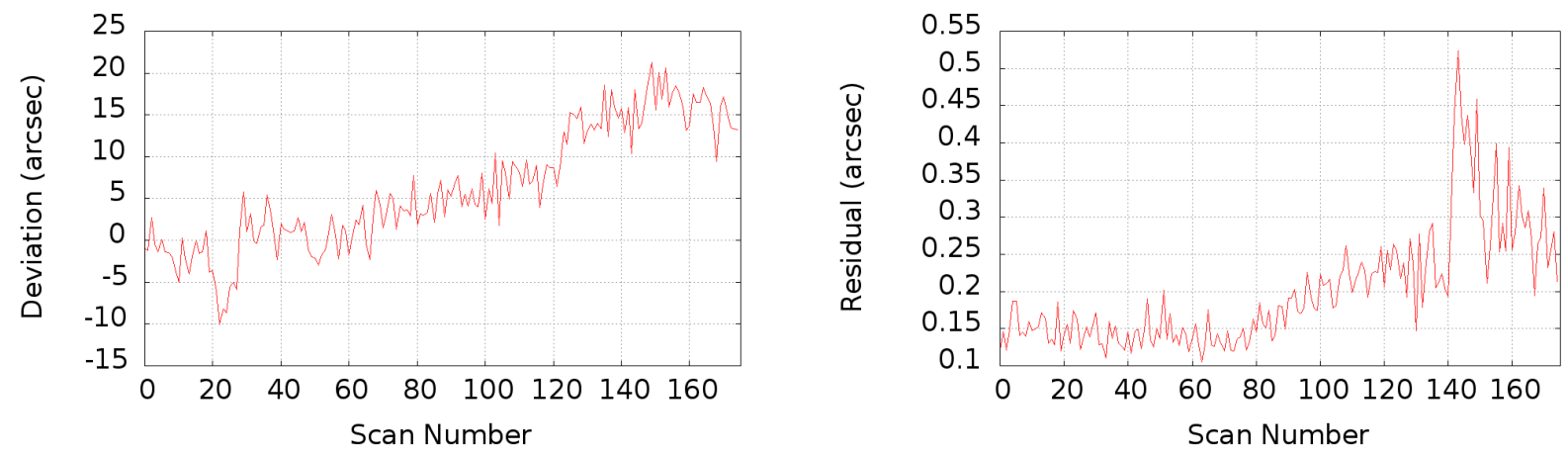

Figure 6. The deviation (left) and RMS residual (right) derived from the linear fits to the scans.

the set-point, and the ambient air temperature outside the enclosure relative to $24^{\circ} \mathrm{C}$ is plotted in figure 7 for a 24 hour period. Part of the response to the step-change in set-point is visible at the left edge of the figure. Data was collected for 100 scans between the 12 and 24 hour marks. The ambient air temperature varied by over $0.4 \mathrm{~K}$ during this interval and the stator temperature was maintained within a $50 \mathrm{mK}$ band around the set-point.

The angle measured by the resolver is plotted against the reticle position in figure 8 . The scans were divided into two groups based on the direction the rotor was slewed immediately after the previous scan. The individual scans show the linear relationship expected and have very little scatter. There is some variation from scan to scan, but the range of angles at a fixed optical reference is less than observed with the two plane configuration. Somewhat unexpectedly there is evidence of hysteresis in the mechanism, as there is a clear distinction between the scans based on the direction of the previous slew. The difference in the mean resolver angle of the two groups is 1.48 arcsec. The RMS of the deviation from the respective means is less than 0.6 arcsec.

A straight line was fitted to the individual scans. The deviation in the resolver angle at a fixed optical reference 


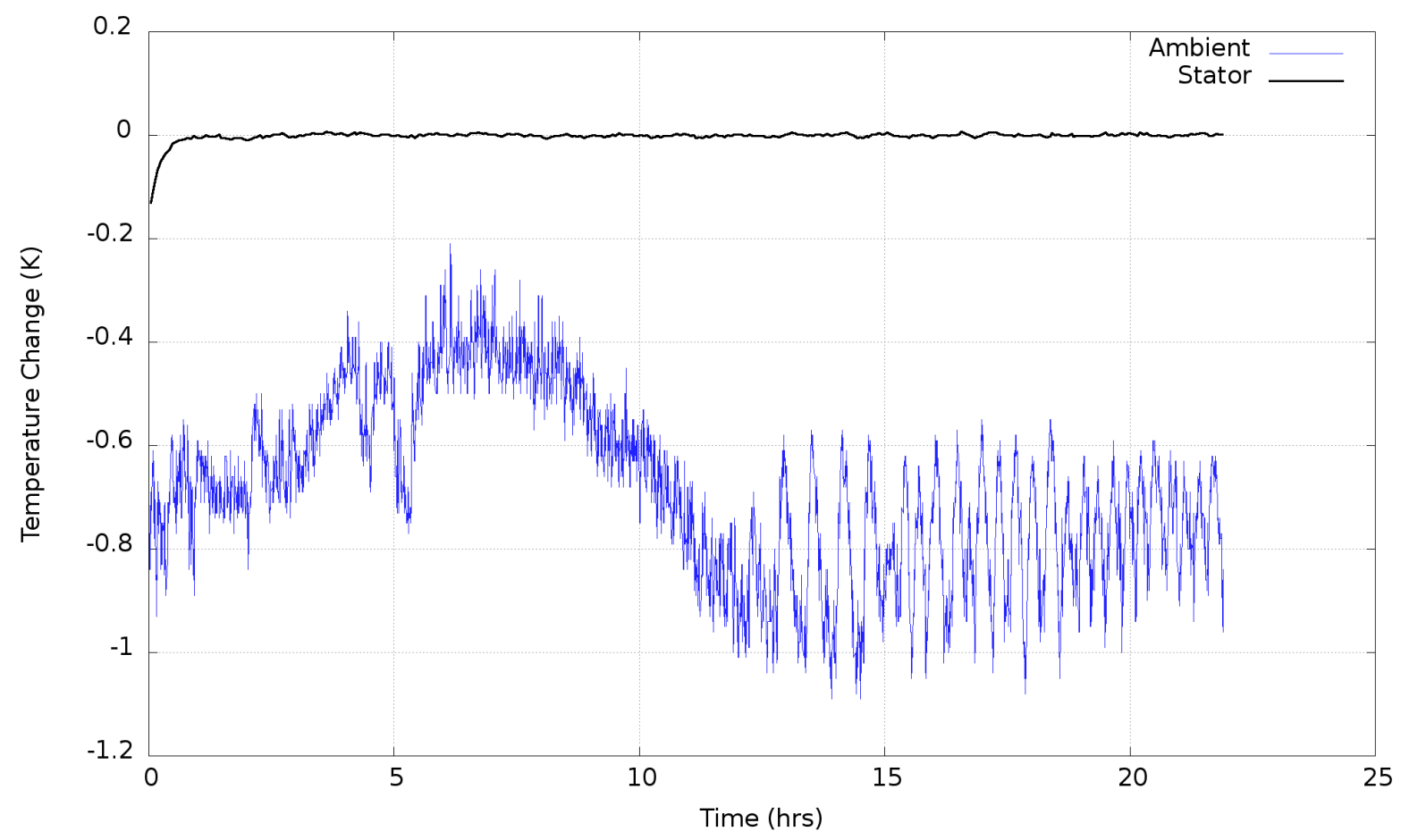

Figure 7. Temperature stability of the stator during the data collection campaign for the one-plane resolver target.

is plotted against the scan number on the left of figure 9. The direction of the previous slew is indicated by the color and shape of the points. The RMS of the residuals is plotted in the panel on the right of figure 9. This clearly shows the hysteresis and a slow drift in the nominal measure of the rotor angle. The RMS of the high-pass filtered resolver angle for the two groups is 0.26 and 0.14 arcsec. The group of scans with the larger variation had slewed immediately after the previous scan in the opposite direction to the scan. Because the rotor is slewed back to an angle just out of the camera field of view the scan is a continuation of relative large rotation. For the other group the rotor changes direction just prior to the scan.

The runout in the Y-direction is plotted against the runout in the $\mathrm{X}$-direction in figure 10 for all 100 scans. The traces are colored according to the direction of the slew prior to the scan, which shows a clear separation of the two groups of scans.

The rotor was configured with the optic supported in the bore of the rotor and the stator was rolled $90^{\circ}$ to face the optic towards the autocollimator. The image row and column directions map to the stator with a $45^{\circ}$ rotation relative to the $\mathrm{X}-\mathrm{Y}$ axes. These axes are label $\mathrm{U}$ and $\mathrm{V}$, and the latter is directed from the rotation center to towards the stepper motor. The plate scale, determined from the scan data-sets, was 0.326 arcsec/pixel. The rotor was stepped through 21 revolutions in $0.75^{\circ}$ increments and the location of the projected reticle was measured in the capture images and converted to tip and tilt in arcseconds. The first 10 revolutions are shown in figure 11. This shows structure in both the $\mathrm{U}$ and $\mathrm{V}$ components on the scale of one revolution and at shorter scales matching the roller-rotor gear ratio. The maximum deviation in the U-direction is larger than in the V-direction. Tip and tilt is expected to be caused by concentricity errors in the rollers, and this difference is consistent with the different baseline lengths in the rotor support points. The maximum deviation of the rotor plane from the Z-axis is just in excess of 1 arcmin. From Eqn (9), it follows that tip and tilt can lead to errors in the measurement of the gaps of up to $3 \mathrm{~nm}$. The corresponding uncertainty in the measured angle is of order one arcseconds.

Figure 12 shows the locus of the tip and tilt from 21 revolutions of the rotor as a continuous line and the first revolution is marked with dots. The cycles on scales of 20 arcsec are caused by the concentricity errors in the 


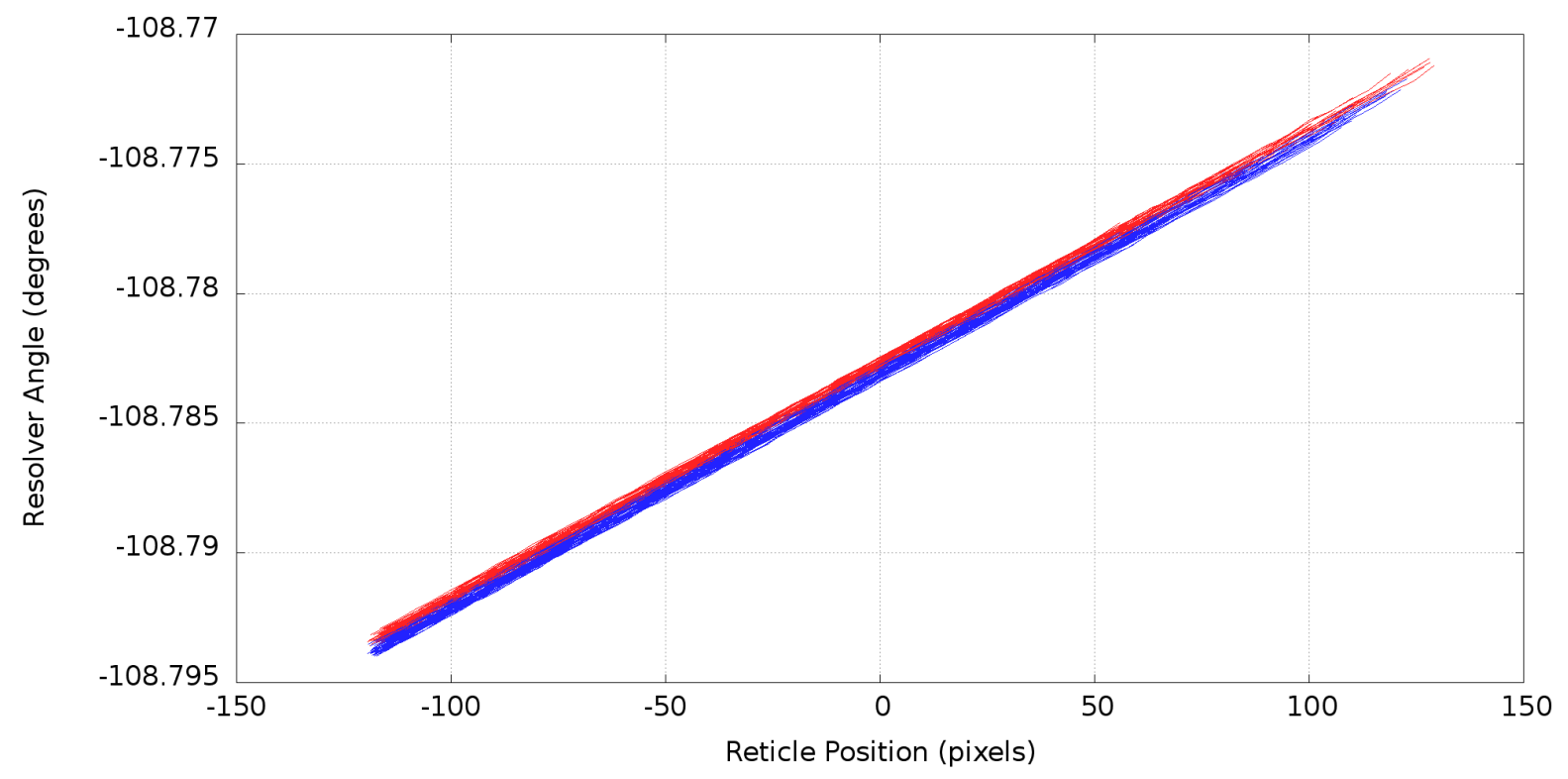

Figure 8. Scans of one-plane resolver with rotor angle near $-108^{\circ}$. Scans are colored based on the direction of the slew that immediately proceeded the previous scan.
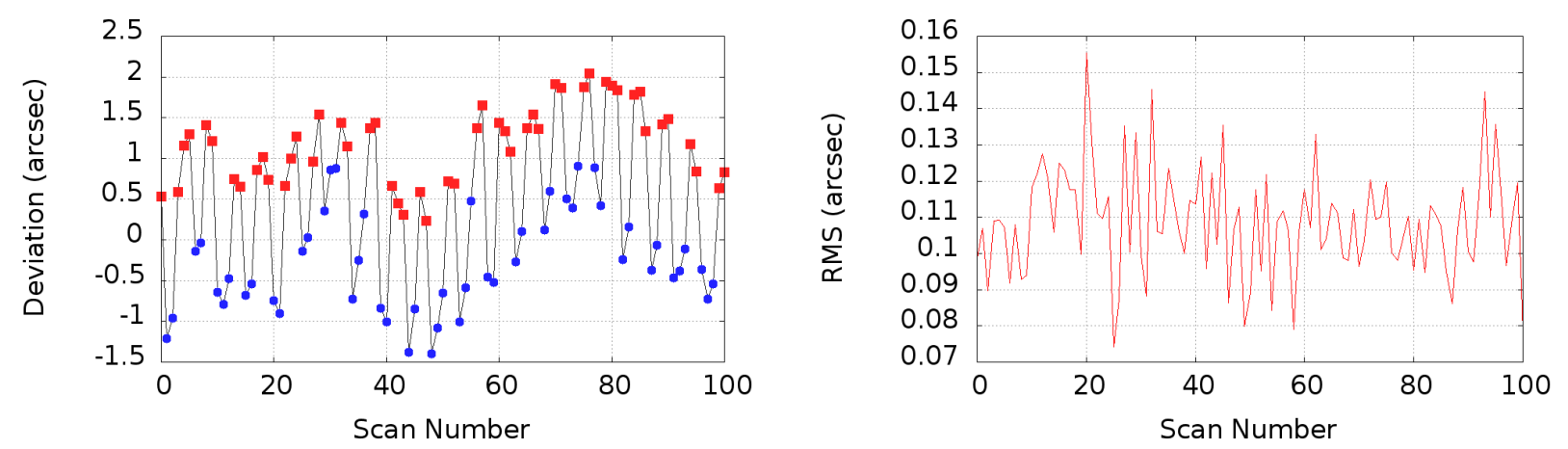

Figure 9. The times series of the angle derived from the one-plane resolver (left) and the RMS of the residuals about the linear fit to the scan (right).

manufacture of the tapered rollers. The large scale structure is cyclic with a period of one revolution, but has a more complex structure. In principle this is the result of superposition of periodic structure in the rotor and static misalignment of the optic from the rotation axis, though the latter would prescribe a circle in figure 12 so there is no evidence to suggest misalignment makes a significant contribution.

\section{DISCUSSION}

The determination of rotor angle from four capacitive sensor measurements was based on the assumption that the sensors measure the distance to the nearest point on the cam of the resolver target. Analysis shows that this assumption leads to inaccurate determination of the absolute rotor angle, but it does not significantly effect the precision nor the runout invariance of the resolver. Non-linearity in the sensor response do degrade the runout cancellation by opposing sensors, but they are dependent on tilt of the target surface and are greatest for small gaps. The resolver geometry does not create this scenario. The two plane resolver target was tested in a poorly temperature controlled environment with the rotor in an orientation that should have been relatively insensitive to the effects of uniform thermal expansion. Drift was observed in the derived rotor angle that was still 


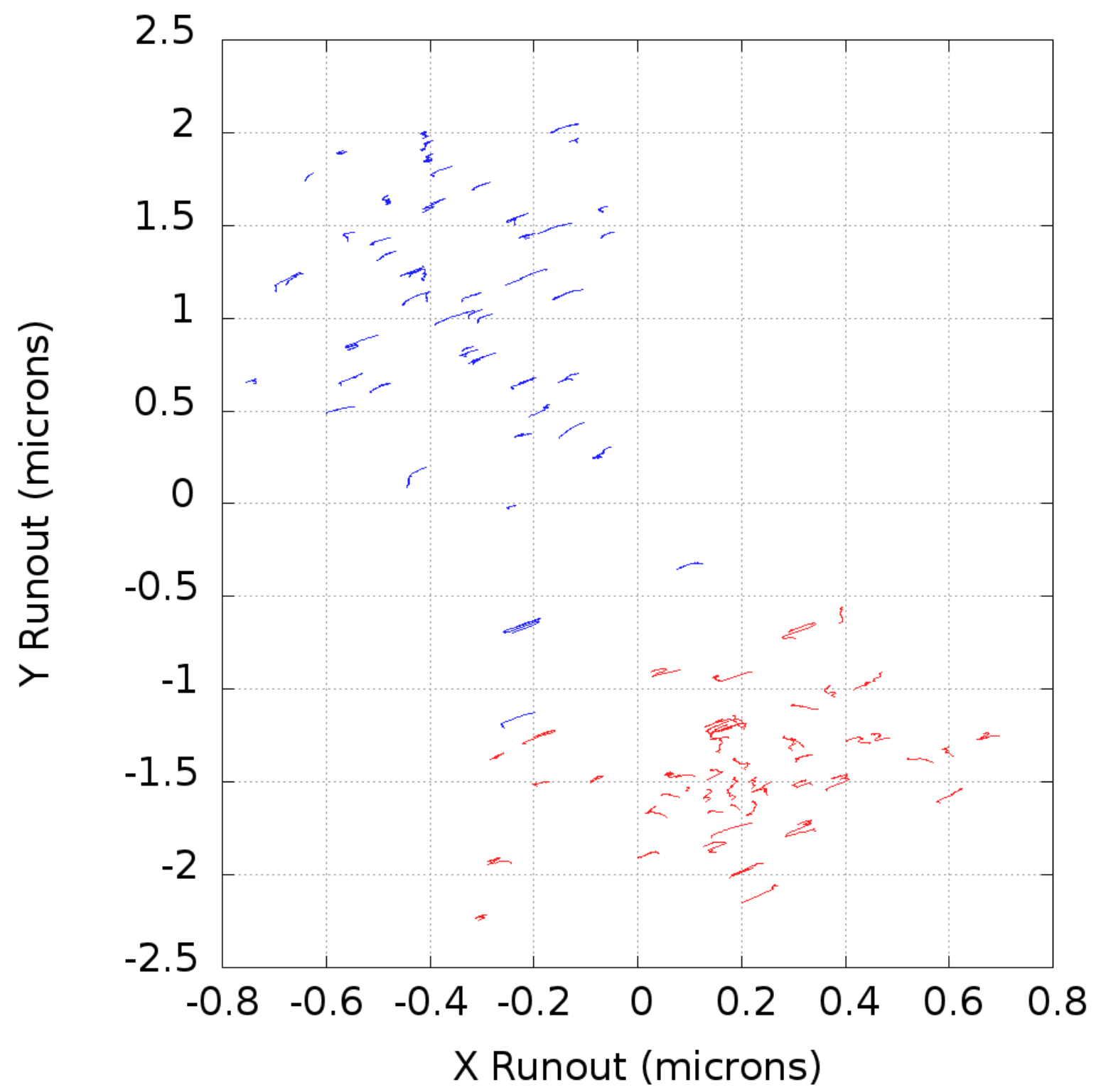

Figure 10. Traces of the location of the rotor center during the scans. The traces are colored according to the direction of the previous slew.

attributed to temperature change. Over short timescales that included a significant slew of the rotary mechanism the repeatability was found to be 1.7 arcsec. With the one-plane resolver and temperature stabilization the repeatability was demonstrated to be 0.14 arcsec when the effects of hysteresis were taken into account. The data required to determine if hysteresis was present for the two-plane resolver target was not gathered, but there is no reason to believe hysteresis is related to the resolver target.

The rim-bearing rotary mechanism was shown to maintain the rotor plane to within \pm 1 arcmin. Concentricity errors in the tapered rollers contributed significantly to the tip and tilt of the rotor, but there were sources of tip-tilt with a period of one revolution. The nominal gear ratio between the rotor and rollers is 11.6. To also attribute the large scale structure to roller concentricity errors would require no slippage between the contact surfaces and a period of five revolutions. It is clear from figure 12 that the cycle defining the large scale tip-tilt 


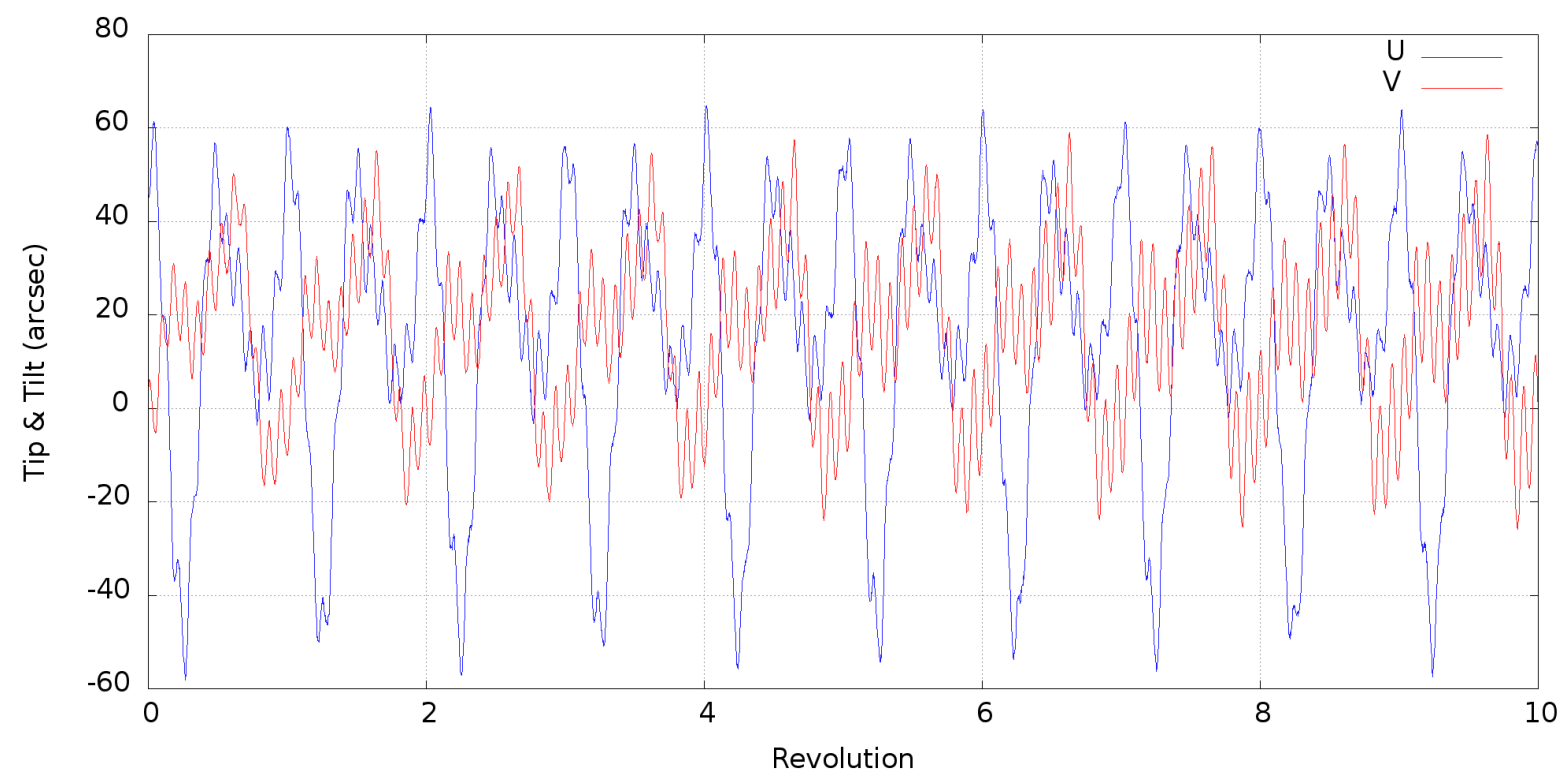

Figure 11. Tip and tilt of the rotor disk as the rotor was turned through approximately 10 revolutions. The $\mathrm{V}$ axis was defined as the center-to-motor direction and the $\mathrm{U}$ axis is perpendicular.

signature is one revolution, not five. Physical structures on that scale may have been caused by distortion of the rotor disk during manufacture, or wear on the conical surfaces during testing. Inspection of the rotor showed a track had been worn in the upper face of the rotor at the nominal contact point with the large end of the tapered rollers. Such a track could induce periodic tip, tilt and runout of the rotor. Note the rotor had been driven through several thousand revolutions prior to measuring the tip-tilt and the surfaces were not lubricated.

Wear in the rotor may also contribute to hysteresis. It indicates there are tracking errors in the rolling surfaces, which can lead to changes in the load via deflection of the flexure hinge. Since the pinch rollers are supported by the same posts that hold two of the sensors, flexure in the stator due to a changing load would make small changes to the sensor locations. In the prototype mechanism a $24 \mathrm{~nm}$ change to all sensor locations driven by flexure in the stator would be equivalent to a change in temperature by $1 \mathrm{~K}$.

\section{CONCLUSION}

One and two-plane resolver target designs have been tested on a rim bearing rotary mechanism that exhibited runout in excess of $10 \mu \mathrm{m}$ and tip-tilt in excess of $1 \mathrm{arcmin}$. With four 24-bit capacitive sensors the resolver precision was shown to be 0.15 arcsec during small moves on short timescales. The one-plane target was shown to be repeatable to this precision after a single non-trivial rotation, but over longer timescales the repeatability degraded to 0.6 arcsec. Whether the drift observed in the angle measured by the resolver was caused by temperature changes less than $50 \mathrm{mK}$ or the accumulative effects of moving the mechanism was not established. The two-plane resolver design was shown to less repeatable than the one plane design, though the test conditions were not identical in both cases. This result was expected, given the two plane design is more susceptible to error from tip-tilt of the resolver target. The mechanism was found to exhibit hysteresis of 1.5 arcsec. The phenomenon that produced the hysteresis was not reliably identified, but the behavior was stable and could easily be incorporated into a motion control strategy. The runout invariant resolver is sensitive to changes in the location of the sensors. Thermal expansion and mechanical strain are both sources of systematic error in the rotor angle. The error is a sinusoidal function of the resolver angle, and is therefore zero at two rotor orientations. This may be exploited in designs that require high precision and discrete set points. 


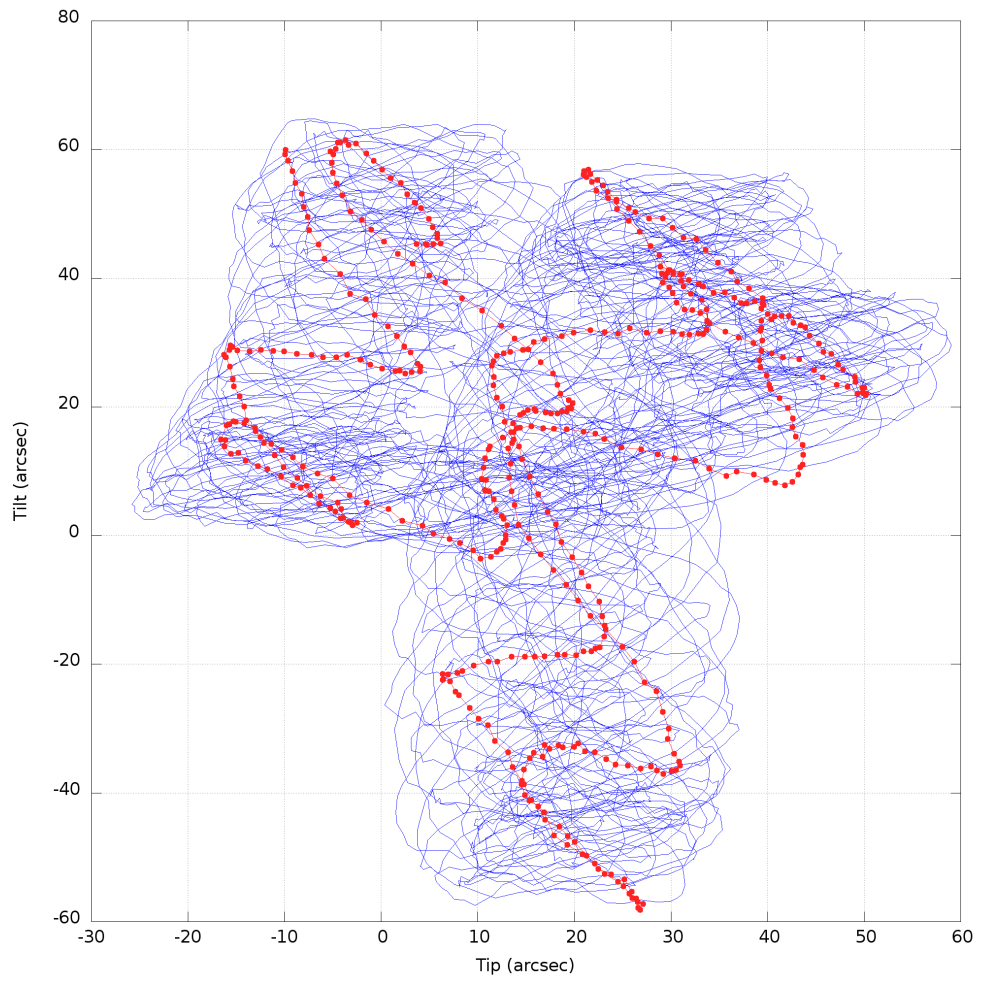

Figure 12. Tip and tilt locus from 21 revolutions of the rotor.

\section{ACKNOWLEDGMENTS}

This work has been undertaken as part of the GMTIFS instrument design and is funded by the Giant Magellan Telescope Organization. We thank them for their ongoing support.

\section{REFERENCES}

[1] McGregor, P. J. et al., "GMT integral-field spectrograph (GMTIFS) conceptual design," Proc. SPIE 8446, $8446-8446-15$ (2012).

[2] Sharp, R. G. et al., "GMTIFS: The Giant Magellan Telescope integral fields spectrograph and imager," Proc. SPIE 9908, 9908 - 9908 - 15 (2016).

[3] Hart, J. et al., "GMTIFS: cryogenic rotary mechanisms for the GMT Integral-Field Spectrograph," Proc. SPIE 9912, 9912 - 9912 - 17 (2016). 